\title{
One-dimensional warm electron transport in GaN quantum well wires at low temperatures
}

\author{
S.K. Sarkar \\ Department of Electronics and Telecommunication Engineering, Jadavpur University, Kolkata-700 032, India \\ e-mail:su_sarkar@hotmail.com
}

Abstract. One-dimensional warm electron coefficient $(\beta)$ and Ohmic mobility $\mu_{0}$ in a square quantum well wire of $\mathrm{GaN}$ are studied for lattice temperatures in the range of $4-10 \mathrm{~K}$, which are important from the application point of view. Electron scattering via deformation potential acoustic, piezoelectric, and ionized impurity are incorporated in the calculations. Carrier distribution is considered to be a heated Fermi-Dirac. Ohmic mobility $\left(\mu_{0)}\right.$ is larger for a quantum well wires of greater width and decreases slowly with the rise of lattice temperature. $\beta$ is negative, and its magnitude falls with increasing lattice temperature and is greater for wider width of the wire. The computed results are discussed in terms of the interplay of the various scattering mechanisms depending upon the model parameters.

Keywords: warm-electron, acoustic, piezoelectric, quantum wires, scattering rates

Paper received 24.03.03; accepted for publication 16.06.03.

\section{Introduction}

In the absence of any electric field, the free carriers in a semiconductor move about at random and in thermal equilibrium with the lattice. Fermi-Dirac distribution function is obeyed by the carriers in degenerate materials. In the presence of an electric field the distribution function undergoes a change since the carriers gain energy from the field. A steady state condition is established when the rate of energy gained from the field equals the rate of energy lost to the lattice through electron-photon interaction.

For low electric fields, when the drift energy of the carriers is a small fraction of the total energy, the solution of the Boltzmann transport equation is achieved by a two-term Legendre polynomial expansion of the distribution function. In the limit of a vanishing electric field the first, i.e. the symmetric part is the equilibrium distribution function appropriate to the lattice temperature. The second term or perturbation term is proportional to the applied field and is controlled by the nature of the scattering mechanisms and the band structure of the material. The carriers may still be considered to be at the lattice temperature, the effect of the field being only to impart an average velocity to the carriers. The drift velocity is proportional to the field so that Ohm's law is valid for semiconductors at vanishingly small fields.

At slightly larger fields, the two-term Legendre polynomial expansion of the distribution function is allowed, since the drift energy can still be much smaller than the total energy. However, the symmetric term is no longer the equilibrium distribution function at the lattice temperature. It will now depend on the applied field, the scattering mechanisms and on the band structure. The electron-electron interaction is stronger for quantum well than in bulk material as modulation doping weakens the ionized impurity scattering. The electron temperature model is hence appropriate in a low dimensional electron gas system. The symmetric term of the distribution function can thus be taken to be Fermi-Dirac characterized by an electron temperature $T_{e}$ different from lattice temperature $T_{L}[1-3]$. The relationship between the current and the field becomes nonlinear, the mobility begins to show a quadratic dependence on the field. This range of fields where deviations from Ohm's law are just beginning to occur is known as the 'Warm electron range'. 


\section{S.K. Sarkar: One-dimensional warm electron transport in ...}

Recent progress in fabrication technique has made it possible to observe quantum effects in semiconductors with effective low-dimensional structures [4-6]. Transport properties of such systems have been extensively investigated in both lateral and vertical structures [7-10]. Such structures are now widely recognized as a very promising basis for future technological applications. Quasione-dimensional (1D) quantum wires (QWRs) were expected to exhibit high electron mobilities at low temperature [11]. One dimensional electron transport is important from physics and application points of view [12].

As suggested by a growing number of authors, $\mathrm{GaN}$ is becoming an important material with regard to the development of opto-electronic devices operating in either the blue region of the visible spectrum or the near ultra violet (UV) regime due to its appropriate bandgap magnitude [13]. GaN has its ability to operate at high power levels and in high temperature environments [14]. Furthermore, large peak of $\mathrm{GaN}$ electron velocity makes it an important candidate for high frequency applications as well. Recently $1 \mu \mathrm{m} \mathrm{AlGaN/GaN}$ heterostructures field effect transistor [HFET] with a cut-off $f_{T}$ of $18.3 \mathrm{GHz}$ has been fabricated [15]. It is anticipated that further improvements in device design will yield even faster devices $[16,17]$. The achievement of blue and green light emitting diodes (LEDs) and blue-light emitting lasers [18], as well as prototypes of several micro electric devices produced from $\mathrm{GaN}$ based materials is intriguing, giving the high density $\left(10^{14} \mathrm{~m}^{-2}\right)$ of dislocations present in most device structures. Substantial defect reduction via the use of lateral overgrowth techniques and substrate removal have resulted in reported life time assessments [19]. This has had a significant impact on the commercial importance of this material [20]. However, the mobility of $\mathrm{GaN}$ is low for bulk material. This leads to the idea that by reducing the size of the material it is possible to enhance the mobility $\left(\mu_{0}\right)$ as it is seen in quantum well (QW) and quantum wire (QWR) of other materials [21]. In view of the above analysis it is very important to study one-dimensional (1D) warm electron transport in GaN quantum wire.

In the present work we calculate the Ohmic mobility $\left(\mu_{0)}\right.$, the warm-electron coefficient $\beta$ and the relaxation rates of the carriers for a degenerate electron distribution for lattice temperature in the range of $4-10 \mathrm{~K}$ which is important for practical application [22]. Carrier scatterings via ionized impurities, acoustic deformation potential and piezoelectric are incorporated in the calculations. We assume a heated drifted Fermi-Dirac distribution for a basic understanding of the warm electron behaviour. We want to investigate the dependence of $\mu_{0}$, and $\beta$ on the width of the quantum well wire (QWR), lattice temperature and carrier concentration. We also study the variation of momentum relaxtion rates on the carrier energy for various lattice temperatures. Dependence of energy loss rate $S\left(T_{L}\right)$ on the lattice temperature and channel length (width) is also studied. We discuss the results in terms of the interplay of the various scattering mechanisms depending upon the model parameters.

\section{Analytical details}

In our calculations, we consider a square quantum wire in which the electron motion is restricted in the longitudinal direction only. The arm of the square cross section of the QWR is referred to as channel length (width). For the system parameter, of the interest here, the separation between the lowest and the next higher sub-band is found to be at least two times higher than the average electron energy. Thus inter sub-band transitions are not significant and hence in an infinite barrier height approximation, the carriers can be considered to occupy the lowest sub-band only. In this preliminary work we take the distribution function $f(\mathbf{k})$ to be a heated Fermi-Dirac:

$f(\mathbf{k})=f(E)+\left(\hbar p_{d} K_{z} / m^{*}\right)\left(-\delta f_{0} / \delta E\right)$

Where $k_{z}\left[=\sqrt{2 m^{*} E / h}\right]$ is the 1D wave vector for energy $E, p_{d}$ is the crystal momentum, $m^{*}$ is the electron effective mass, $h$ is Planck's constant divided by $2 \pi$, and $f_{0}(E)$ is the Fermi-Dirac distribution function with an electron temperature $T_{e}$.

The heated Fermi-Dirac distribution function model employed here is strictly valid when electron-electron interactions dominate in the sharing of energy and momentum. In the bulk material, electron-electron scattering is not dominating because the prevailing ionized impurity scattering is of comparable strength. In 1D systems, the effect of the ionized impurity is suppressed due to modulation doping. But here electron-electron scattering leads to an interchange of particles so that the actual distribution differs from the form given by Eq. (1). However, we are not interested in the exact shape of the distribution function. Rather we wish to determine the macroscopic parameters, which involve an average over the distribution function. Fortunately, these parameters are not much sensitive to the exact nature of the distribution function. In fact, the electron temperature model adopted here does not lead to seriously inaccurate results [23]. Our model takes less computing time and is free from the statistical fluctuations of a numerical Monte Carlo simulation. It can thus be used to have a feeling of the effects of the charges of various system parameters on the Ohmic mobility, relaxation rates and warm electron transport coefficient $\beta$.

In a rectangular cartesian frame, we take $x$ and $y$ direction along the two adjacent sides of the cross section of the quantum wire and $z$ direction along the axis of the wire. The momentum relaxation times $\tau_{a c}$ and $\tau_{p z}$ for deformation potential acoustic and piezoelectric scattering, respectively and the energy loss rate $\langle d E / d t\rangle_{a c}$ and $<d E / d t\rangle_{p z}$ for these two scattering processes are found from the square of the metrics element for the $1 \mathrm{D}$ system [24]. In the energy loss rate, the terms up to the linear in $\left(T_{e}-T_{L}\right)$ are kept, $T_{L}$ being the lattice temperature, because these are only important term in the warm electron regime. The nonequipartition of the acoustic phonons is considered in the calculations. 
The momentum relaxation time $\tau_{m}$ for background ionized impurity scattering is calculated following Lee and Vassell [25], for a typical background impurity concentration of $6 \cdot 10^{21} \mathrm{~m}^{-3}$. The effect of the remote impurity is small for a thick undoped spacer in modulation doped structures and so it is not considered here [12].

The electron mobility is given by the expression:

$$
\mu=\frac{2 e}{\pi h n_{1 D}}\left(\frac{2}{m^{*}}\right)_{0}^{\Pi} E^{\frac{1}{2}} \tau\left(-\frac{d f_{0}}{d E}\right) d E
$$

Where e is the electronic charge and $\tau^{-1}=\tau_{a c}^{-1}+\tau_{p z}^{-1}$.

For an applied electric field $F$, the warm electron mobility is

$\mu=\mu_{0}(1+\beta F)$

We expand $\mu$ in a Taylor series in $\left(T_{e}-T_{L}\right)$ and keep only the first order terms in $\left(T_{e}-T_{L}\right)$ as the contribution of the higher order terms are insignificant [22]. Thus we get

$\mu=\mu_{0}+\left.\left(T e-T_{L}\right) \frac{d \mu}{d T e}\right|_{T e=T_{L}}$

The balance of energy relationship is

$e \mu_{0} F^{2}=-\left\langle\frac{d E}{d t}\right\rangle_{a c}-\left\langle\frac{d E}{d t}\right\rangle_{p z}=\left(T_{e}-T_{L}\right) S\left(T_{L}\right)$

where the function $S\left(T_{L}\right)$ is obtained numerically from the energy loss rates [22,24].

From Eqs (2), (3) and (4) the warm-electron coefficient $\beta$ is found to be

$$
\beta=\left.\frac{e}{S\left(T_{L}\right)} \frac{d \mu}{d T_{e}}\right|_{T_{e}=T_{L}}
$$

with $\left.\frac{d \mu}{d T_{e}}\right|_{T_{e}=T_{L}}$ being obtained from Eq. (1).

\section{Numerical results and discussions}

Calculations are done for a quantum wire of $\mathrm{GaN}$ with the material parameters given in the Table 1. Fig. 1 shows the variations of relaxation rates for the various scattering mechanisms vs the electron energy for a typical channel length of $10 \mathrm{~nm}$ and carrier concentration of $6 \cdot 10^{7} \mathrm{~m}^{-1}$. The relaxation rates decreases with the increasing energy of the electron. The phenomenon is attributed due to the fact that the density of states in the quantum wire (QWs) varies as $E^{-1 / 2}$. The relaxation rates are higher at lower lattice temperature. The ionized impurity concentration is taken as $6 \cdot 10^{21} \mathrm{~m}^{-3}$.

The dependence of Ohmic mobility $\left(\mu_{0}\right)$ with carrier concentration for various lattice temperatures $(4,6$, and $8 \mathrm{~K}$ ) is represented in the Fig. 2. The mobility is found to

\section{Table 1. Material parameters of GaN}

\begin{tabular}{ll}
\hline \hline Parameters & Value \\
\hline Electron effective mass, $m^{*}(\mathrm{~kg})$ & $0.22 \times 9.1 \times 10^{-31}$ \\
\hline Mass density, $\rho\left(\mathrm{kg} / \mathrm{m}^{3}\right)$ & $6.15 \times 10^{3}$ \\
\hline Longitudinal sound velocity, $u_{l}(\mathrm{~m} / \mathrm{s})$ & $5 \times 10^{3}$ \\
\hline Transverse sound velocity, $u_{t}(\mathrm{~m} / \mathrm{s})$ & $5 \times 10^{3}$ \\
\hline Acoustic deformation potential, $E_{l}(\mathrm{~J})$ & $8.3 \times 1.602 \times 10^{-19}$ \\
\hline Static dielectric constant, $K_{s}$ & 10.4 \\
\hline Optic dielectric constant, $K_{\infty}$ & 9.5 \\
\hline LO phonon energy $\hbar \omega(\mathrm{eV})$ & 0.093 \\
\hline
\end{tabular}

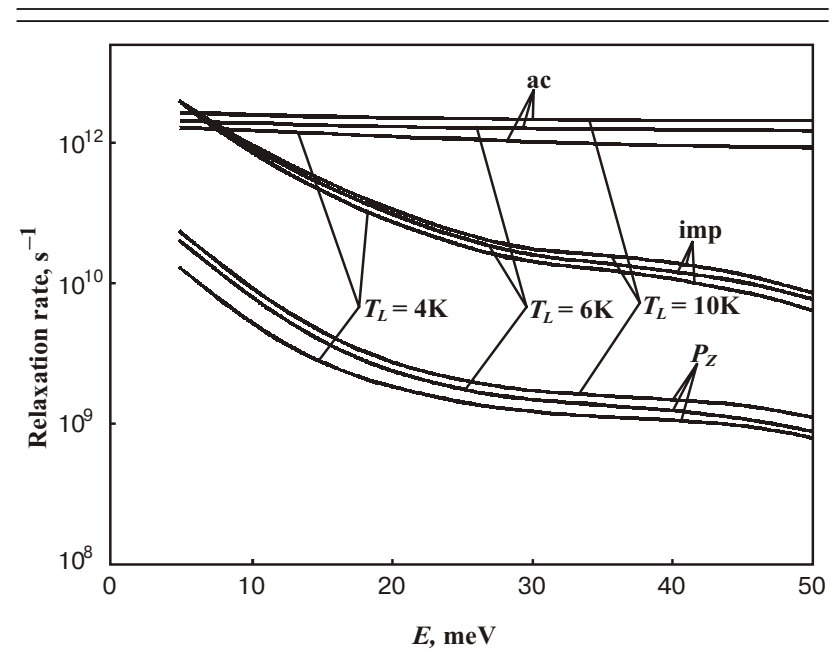

Fig. 1. Variation of momentum relaxation rates versus electron energy for deformation potential acoustic (ac), piezoelectric (pz), ionized impurity (imp) for GaN square quantum wires. For each type of scattering mechanism, relaxation rates are plotted for three different lattice temperature $(4,6,10 \mathrm{~K})$ at a typical channel length (width) of $10 \mathrm{~nm}$ and carrier concentration of $6 \times 10^{7} \mathrm{~m}^{-1}$.

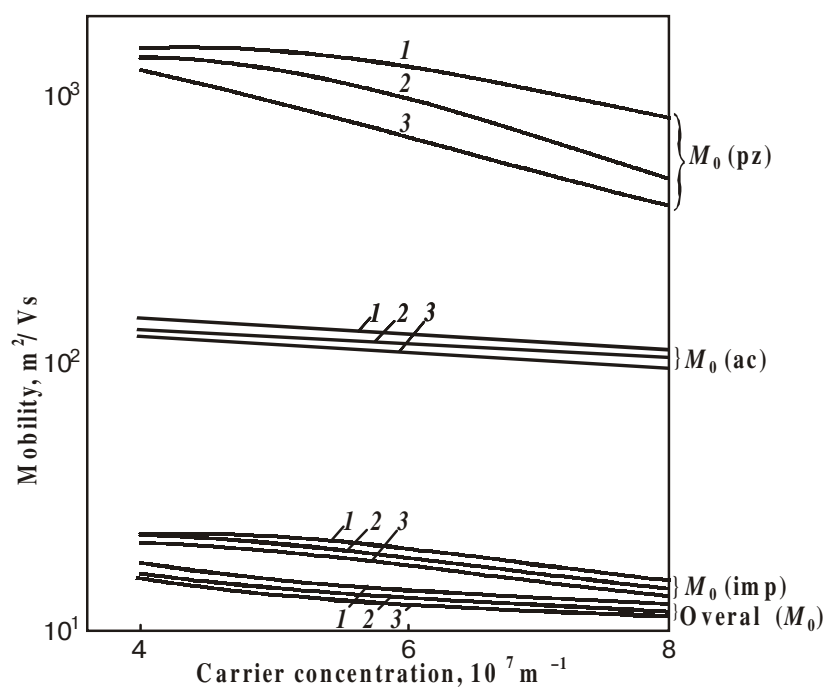

Fig. 2. Plots of Ohmic mobility $\left(\mu_{0}\right)$ with carrier concentration for different values of lattice temperatures for a typical channel length of $10 \mathrm{~nm}$. The other relevant parameters are the same as in Fig. 1.

SQO, 6(2), 2003 


\section{S.K. Sarkar: One-dimensional warm electron transport in ...}

decrease slightly with increasing carrier concentration. The combined scattering mechanisms and the upward shift of the Fermi-level at higher carrier concentrations account for such variation of mobility $[5,26]$.

Fig. 3 depicts the variation of Ohmic mobility $\left(\mu_{0}\right)$ with lattice temperature for different values of channel length. The other parameters are the same as in Fig. 1.The mobility is found to decrease slightly with increasing lattice temperature. The electrons move to the higher energy states at higher lattice temperature. The decrease of the density of the states at high energies overrides the increase of the momentum relaxation time at such energies causing the mobility to decreases with a rise in lattice temperature [22]. It is found that $\mu_{0}$ increases with the increasing channel length. This can be attributed by the reduced scattering at higher channel length [26].

Fig. 4 shows the variation of $S\left(T_{L}\right)$ with the lattice temperature for various values of channel width. The other parameters are the same as in Fig. 1. As the lattice temperature increases, the phonon occupation number increases, leading to an increase of the energy loss rate. So the function $S\left(T_{L}\right)$ is found to increase with the lattice temperature as evident from the Fig. 4. Energy loss rate is found lower at higher channel length. It is also revealed from the Fig. 4 that the deformation potential acoustic scatterings dominate the energy loss rate. Piezoelectric contribution is at least an order of magnitude lower under identical condition.

The dependence of warm electron coefficient $\beta$ on lattice temperature is represented in Fig. 5 for various values of channel length. $\beta$ is negative here as $\left.\frac{d \mu}{d T_{e}}\right|_{T_{e}=T_{L}}$ is negative. The Fig. 5 depicts that the magnitude of $\beta$ decreases with an increment of lattice temperature. The magnitude of $\beta$ is enhanced at higher value of the channel length.

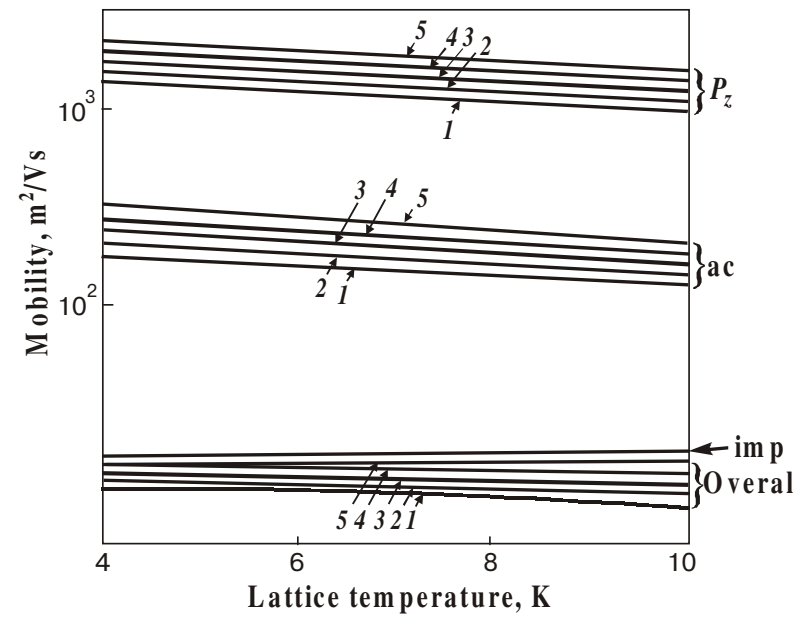

Fig. 3. Variation of Ohmic mobility $\left(\mu_{0}\right)$ with lattice temperature for deformation potential acoustic (ac), piezoelectric (pz), ionized impurity (imp) and overall mobility. Curves 1 through 5 are for channel lengths of $8,9,10,11,12 \mathrm{~nm}$ respectively. The other relevant parameters are the same as in Fig. 1.

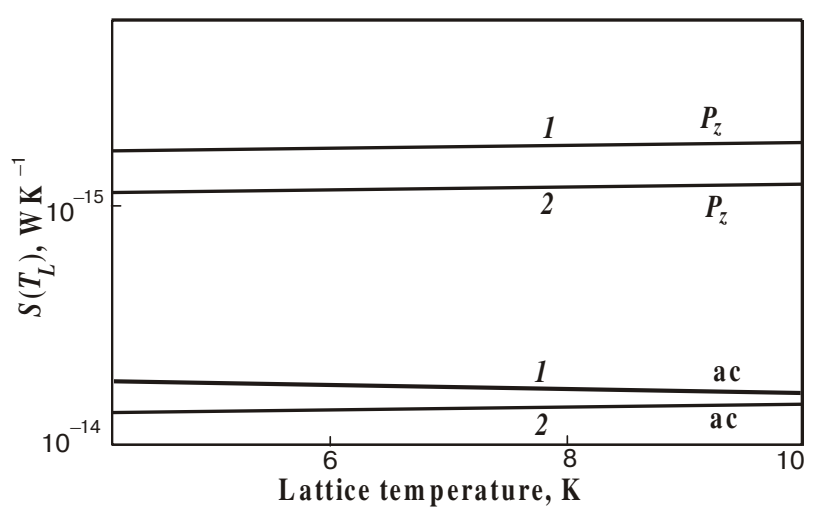

Fig. 4. Dependence of $S\left(T_{L}\right)$ on lattice temperature for various values of channel length (width). Curves 1 and 2 are for the channel lengths of $12 \mathrm{~nm}$ and $8 \mathrm{~nm}$, respectively. The other relevant parameters are the same as in Fig. 1.

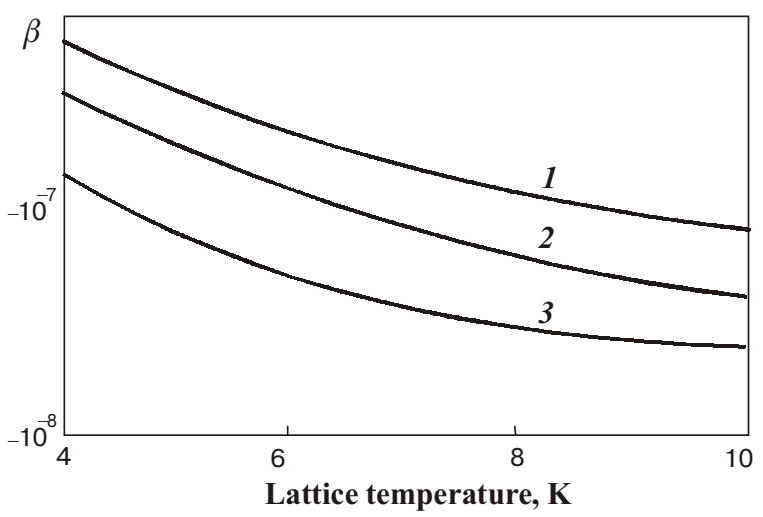

Fig. 5. Plots of warm electron coefficient $\beta$ with lattice temperature for channel length of $12(1), 8$ (2) and $6 \mathrm{~nm}$ (3). The other relevant parameters are the same as in Fig. 1.

\section{Conclusion}

The warm electron effect is observable at fields much lower than $1 / \beta$. Experimental data on $\beta$ for square quantum wire of $\mathrm{GaN}$ are scarce in the literature. Experimental results to square wires are required for a comparison with the present calculations and to test the size dependence of the warm electron transport. The electron mobility in Ohmic and non-Ohmic regimes is a key parameter in characterizing the sample for devices. The present model will surely provide valuable informations and may be utilized during actual applications.

\section{Acknowledgement}

The author thankfully acknowledges the financial support available from University Grants Commission vide order no: F.14-32/2000 dated 12.10.2000. 


\section{S.K. Sarkar: One-dimensional warm electron transport in ...}

\section{References}

1. S.K.Sarkar and D. Chattopadhyay: Solid State Communication 109, 13(1999).

2. S.K.Sarkar and D.Chattopadhyay: Ind. J. Pure \&Appl. Phys. 36,149(1998).

3. S.K.Sarkar and D.Chattopadhyay: Proc. DAE, SSPS, C 40,197(1997).

4. T. Itoh,N.Sano and A.Yoshii: Phys Rev. B 47, 16601 (1993)

5. P.K.Ghosh, S.K.Sarkar and D.Chattopadhyay:Phys. Stat. Sol. (a) 156,365(1996).

6. S.K.Sarkar and D.Chattopadhyay: Nanotechnology. 9, 321(1998).

7. A.T.Johnson et al: Phys. Rev. Lett. 69, 1592 (1992).

8. S.Taracha, Y.Yokura and Y.Hiragama: Phys. Rev. B. 44, 13815 (1991).

9. J.Nishizawa, P.Plotka and T.Kurabayashi: IEEE, ED, 49, 1102 (2002).

10. P.Lei etal: IEEE, ED. 49, 1129(2002).

11. Mickivicins and V.Mitin: Phys. Rev. B. 48,17194 (1992).
12. S.K.Sarkar and D. Chattopadhyay: Physica B. 222,217222(1996).

13. J.G. Kim, A.C. Frenkel, H. Liu and R.M. Park: Appl. Phys., 65,4(1994)

14. B.E. Foutz, L.F.Eastman,U.V.Bhapkar and M.S.Shur: IEEE ED Lett. 17, 354 (1996).

15. M.A. Khan, et al, IEEE Ed Lett, 17, 354 (1996).

16. M.A.Khan et al: Electron Lett. 32, 357 (1996).

17. M.A. Khan, et al: IEEE Lett. 17, 584 (1996).

18. C.M.balkas et al: Journal of Crystal growth 208, 100(2000).

19. S. Nakamura et al: Appl. Phys. Lett. 72(1998) 211.

20. M. Meyer, Compound Semicond. 3 (1997) 8.

21. S. K. Sarkar and D. Chattopadhyay: Phys Rev. B 62, 15331 (2000).

22. D. Chattopadhyay and P.K. Ghosh: J. Appl. Phys. 75, 1 (1994).

23. D.Chattopadhyay: Appl. Phys. A 53, 35(1991).

24. A. Kabasi and D. Chattopadhyay: Solid State Commun. 75, 71 (1996).

25. J. Lee and M.O. Vassell: J. Phys C 17, 2525 (1984).

26. S.K. Sarkar, P.K.Ghosh, D. Chattopadhaya: J. Appl. Phys. 78, 283 (1995) 